\title{
Trechus montiscrystalli Casale, 1979 (=Trechus pecoudi Jeannel, 1937 nec T. brucki pecoudi Colas \& Gaudin, 1935), nuovo sinonimo di Trechus italicus K. Daniel \& J. Daniel, 1898 (=T. samnis Jeannel, 1921) (Coleoptera: Carabidae)
}

\begin{abstract}
Riassunto: A seguito dell'esame delle caratteristiche esterne e genitali di un paratipo maschio, Trechus montiscrystalli Casale, 1979 (nome nuovo proposto per Trechus pecoudi Jeannel, 1937, nec T. brucki pecoudi Colas \& Gaudin, 1935), descritto delle Alpi orientali (Dolomiti, Monte Cristallo), è riconosciuto come sinonimo junior di Trechus italicus K. Daniel \& J. Daniel, 1898 (=T. samnis Jeannel, 1921), specie localizzata in alcuni massicci montuosi dell'Appennino centrale (Abruzzo, Italia). La sinonimia è spiegabile per la località errata riportata nel materiale tipico del taxon suddetto, e tale ipotesi è confermata dalla presenza, in Collezione Pécoud (MNHN), di esemplari di Carabidi raccolti da Pécoud stesso in Abruzzo (Gran Sasso d'Italia) nelle stesse date riportate per Trechus pecoudi Jeannel.

Abstract: Trechus montiscrystalli Casale, 1979 (=Trechus pecoudi Jeannel, 1937 nec T. brucki pecoudi Colas \& Gaudin, 1935), new synonym of Trechus italicus K. Daniel \& J. Daniel, 1898 (=T. samnis Jeannel, 1921) (Coleoptera, Carabidae).

From the examination of both external and genitalia features of a male paratype, Trechus montiscrystalli Casale, 1979 (new name for Trechus pecoudi Jeannel, 1937, nec T. brucki pecoudi Colas \& Gaudin, 1933), described from Eastern Alps (Dolomites, Cristallo Mt.), owing to wrong locality labels is recognized as a junior synonym of Trechus italicus K. Daniel \& J. Daniel, 1898 (=T. samnis Jeannel, 1921), species endemic to some montane massifs of Central Apennine (Abruzzi, Italy). This hypothesis has been confirmed by the presence, in the Pecoud's collection (MNHN), of carabid specimens from Abruzzi (Gran Sasso d'Italia) collected by himself on the same dates as those reported for Trechus pecoudi Jeannel.
\end{abstract}

Key words: Coleoptera, Carabidae, Trechinae, Trechus montiscrystalli, Trechus italicus, synonymic note, Italy.

\section{INTRODUZIONE}

Successivamente alla pubblicazione dell'immensa Monografia dei Trechini mondiali, Jeannel (1937) descrisse Trechus pecoudi su due individui di sesso maschile, con località tipica Monte Cristallo (Alpi del Cadore, Dolomiti orientali), specie ritenuta dall'autore francese affine a T. strasseri Ganglbauer, 1891, presente nelle Alpi Lepontine (Piemonte e Canton Ticino). Tale nome si è mantenuto per anni nei cataloghi faunistici italiani (cfr. Magistretti, 1965).

Casale (1979), nel corso della preparazione di un catalogo dei Trechini e Trechodini mondiali (Casale \& Laneyrie, 1982), rilevando l'omonimia primaria con Trechus brucki ssp. pecoudi, sottospecie descritta da Colas \& Gaudin (1935) del Pic d'Orhy (Pirenei occidentali), in seguito considerata specie distinta e politipica (cfr. Casale \& Laneyrie, 1982; Löbl \& Smetana, 2003; Lorenz, 2005), ma nuovamente ritenuta sottospecie di brucki da Coulon et al. (2011), propose il nome nuovo Trechus montiscrystalli per il taxon alpino suddetto, nome che pure si è mantenuto nei vari cataloghi e nelle checklist fino ai giorni nostri (cfr. Löbl \& Smetana, 2003; Vigna Taglianti, 2005; Casale \& Vigna Taglianti, 2005; Lorenz, 2005; Casale et al., 2006).
Incuriositi dal fatto che nessuno, nei decenni successivi alla descrizione del taxon da parte di Jeannel, fosse stato in grado di ritrovare sul Monte Cristallo o in località prossime una specie del genere Trechus con i caratteri evidenziati dall'autore francese, pur essendo l'area ben nota e frequentata da numerosi entomologi, uno degli autori del presente contributo (AC), durante un periodo di studio a Parigi (Novembre 2012), ha proceduto alla ricerca del materiale tipico conservato in MNHN.

L'olotipo non è stato rintracciato, ma un paratipo maschio, indicato da Jeannel nella descrizione originale, è risultato presente e in perfette condizioni nella monumentale Collezione Georges Pécoud. L'esame del medesimo, e la sua dissezione, hanno dato il risultato sorprendente qui illustrato.

\section{Materiali e Metodi}

Materiale tiPICo esaminato. Trechus pecoudi Jeannel, 1937, 1 paratypus $\widehat{\partial}$ con indicazioni: "Alpes $\mathrm{Ca}$ doriques Monte Cristallo Pècoud (sic) 7.1923", "Pecoudi n. sp. Jeannel det.», «COTYPUS», in MNHN (coll. Pécoud) (Figg. 1-7).

Materiale NON TIPICO ESAMINATO. Trechus italicus K. 


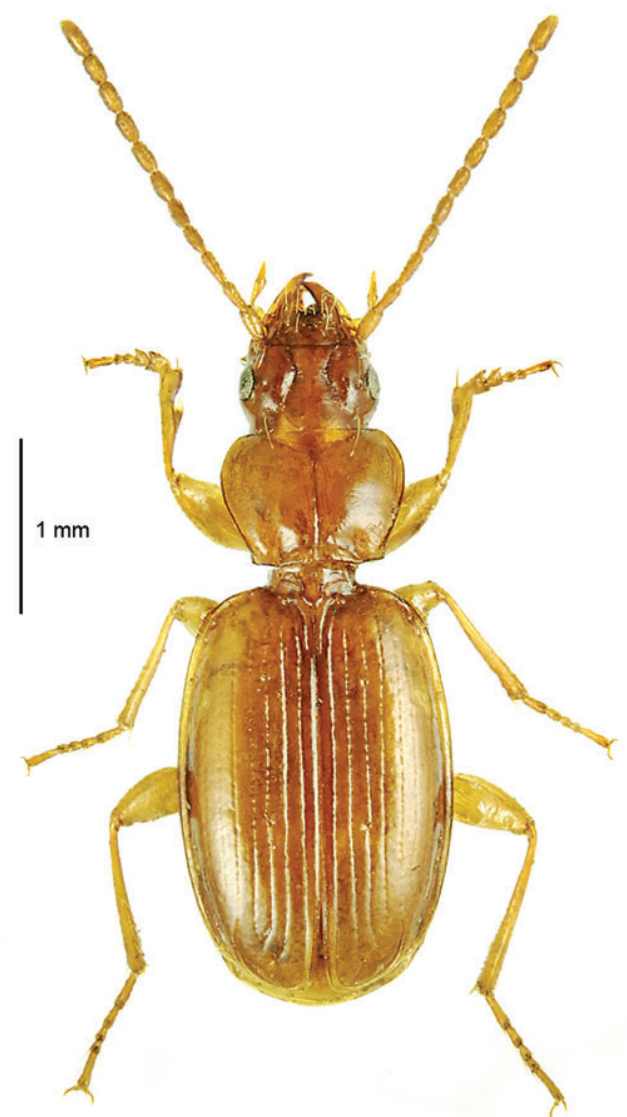

Fig. 1. Trechus pecoudi Jeannel, 1937 (nec pecoudi Colas \& Gaudin, 1935), paratypus maschio (MNHN, Coll. Pécoud): habitus.
Daniel \& J. Daniel, 1898 (=T. samnis Jeannel, 1921) 7 ㅊํ, 우, Abruzzo (L'Aquila), Gran Sasso d'Italia, Rifugio Duca degli Abruzzi, m 2300 s.l.m.,

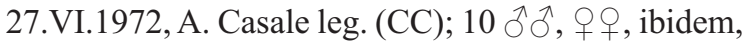
Rifugio Franchetti, m 2400 s.1.m., 18.VII.1976, A. Ca-

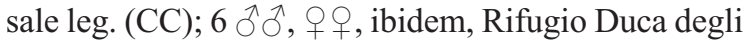
Abruzzi, m 2300, 27.VI.1984, P. Magrini leg. (CM) (Figg. 8,9); 1 ô, Abruzzo (L'Aquila), Gran Sasso d'Italia, m 2500 s.1.m. 19.VI.2002, A. Degiovanni leg. (CD) (Fig. 10); 1 त, Abruzzo (L'Aquila), Campo Imperatore m 2000 s.l.m., , 15.VII.1976, Franzini leg. (CM); 1 q, ibidem, 29.VI.1982, L. Diotti leg. (CM); 1 ô, Abruzzo, Gran Sasso, Campo Pericoli, VIII.1957, E. Laudanna leg. (AVT); $14 \hat{\jmath} \widehat{\jmath}$,, $o$, ibidem, $\mathrm{m} 2500$, Campo Pericoli, 4.VIII.1968, V. Vomero leg. (AVT); 1 ふै, ibidem, 2.IX.1979, M. Zapparoli leg. (AVT); 3

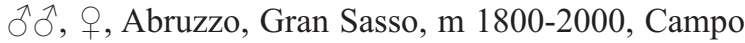

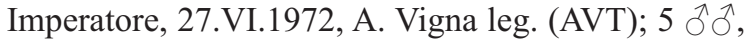
우, Gran Sasso, $m$ 2400, Monte Camicia, 24.VII.1961, F. Cassola, G. Papini leg. (AVT); 1 ว’, Gran Sasso, Monte Portella, 11.VII.1977, G. Narducci

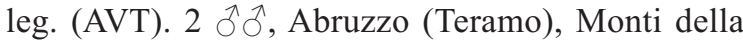
Laga, Monte Gorzano, m 2200 s.1.m., 21.V.1989, P. Magrini leg. (CM) (Figg. 11,12); 1 त, ibidem, 5.V.1983, I. Gudenzi leg. (CM); 1 ふૈ, ibidem, 21.V.1989, A. Pennisi leg. (CM); 1 ふै, Abruzzo, Monti della Laga, Monte Gorzano, m 2455, 5.IX.1968, B.

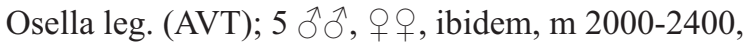
25.VII.1969, B. Osella leg. (AVT). $2 \lambda \hat{\jmath}$, Abruzzo (L'Aquila), Monte Velino m 2200 s.l.m., 20.V.1989,

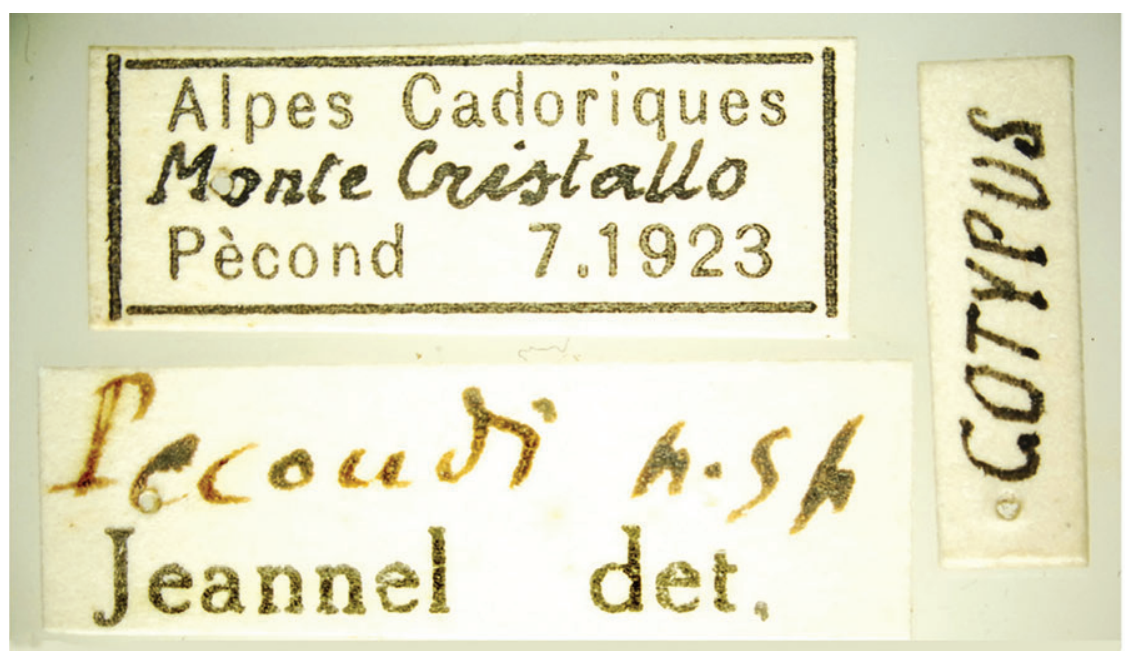

Fig. 2. Cartellini originali allegati al paratipo di Trechus pecoudi Jeannel, 1937 (MNHN). 


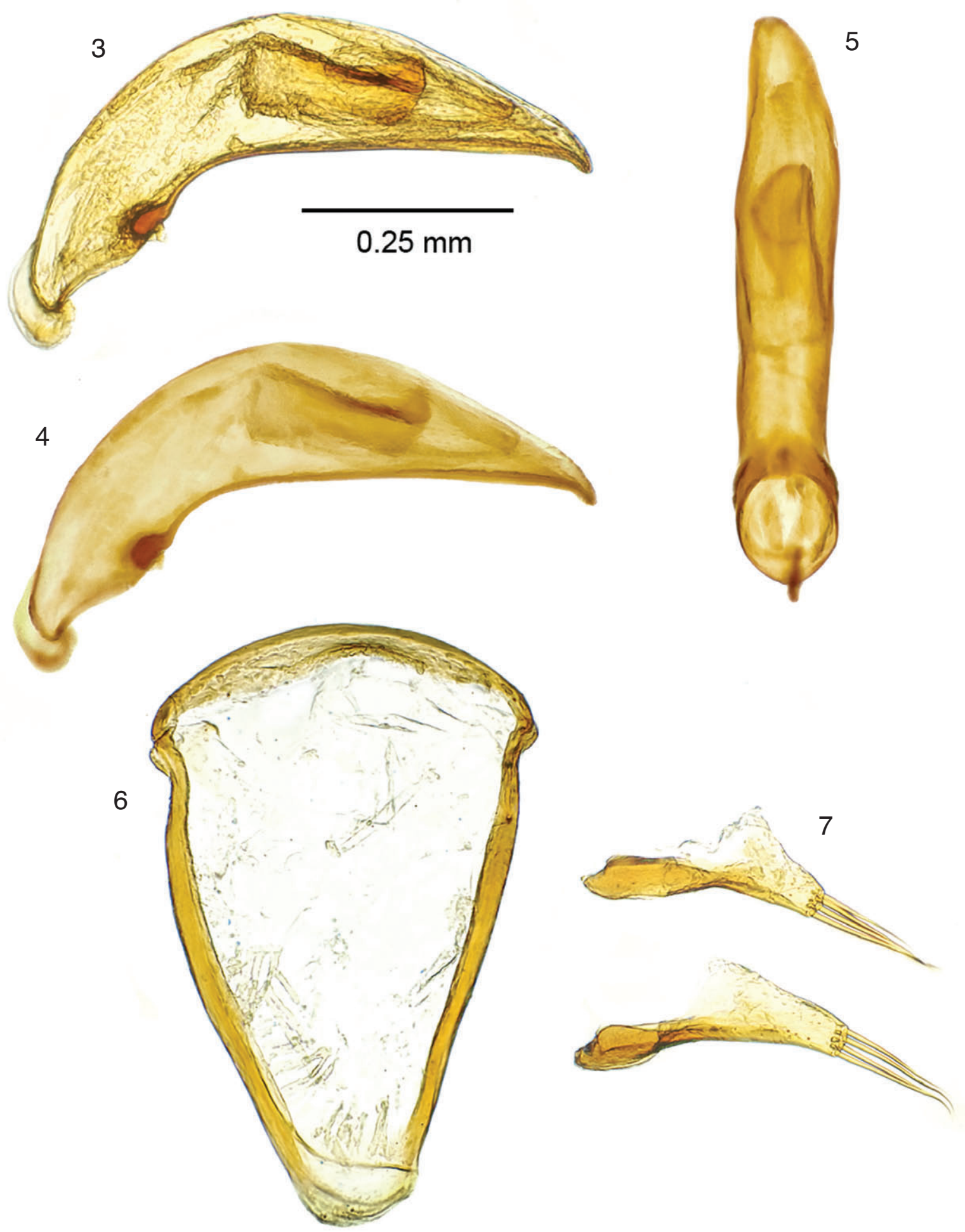

Figg. 3-7. Trechus pecoudi Jeannel, 1937, paratipo đ̂. 3, Edeago in visione laterale su acetato; 4, edeago in visione laterale su perspex; 5, edeago in visione ventrale su perspex; 6, urite IX; 7, parameri. 
P. Magrini leg. (CM); 10 $\widehat{\jmath}, \circ+++$, Abruzzo, Monte Velino, m 2050, 11.VII.1982, S. Bruschi leg. (AVT). 1 ô, Abruzzo, Monte Sirente, 16.VIII.1984, G. Di Giulio leg. (AVT). 1 o, Abruzzo, Monte Marsicano, 26.VII.1933, Santercole leg. (AVT); 20 숭, 우우, ibidem, cresta Nord, m 2200, 10.VIII.1969, A. Vigna leg.

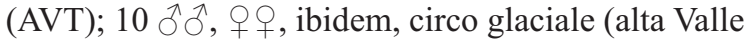
Orsara), m 2050, 21.VII.1973, A. Vigna leg. (AVT). 6 ổ, 우우, Abruzzo, Serra Monte Cappella, nevai Sella Orsara, m 1950, 10.VIII.1969, A. Vigna leg. (AVT).

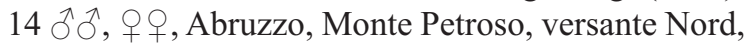
m 2100, 8.VIII.1973, A. Vigna leg. (AVT). 70 ふ઼ 웅, Abruzzo, Monte Meta, nevaio Biscurri, m 1900, 7.VIII.1969, A. Vigna leg. (AVT); $3 \hat{\jmath} \hat{\jmath}$, 우, ibidem, 17.VII.1973, A. Vigna leg. (AVT); $47 \hat{\delta} \widehat{\partial}$, 우우, Monte Meta, m 2240, 7.VIII.1969, A. Vigna leg. (AVT). 2 우오, Abruzzo, Monte Greco, m 2100, 16.VI.1973, E. Colonnelli leg. (AVT); 1 ภ,, , ibidem, m 2100-2280, 27.VII.1973, A. Vigna leg. (AVT); 1 , , ibidem, G. Pa-

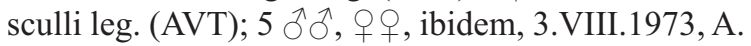
Vigna leg. (AVT).

\section{ACRONIMI DELLE COLLEZIONI}

MNHN: Muséum National d'Histoire Naturelle, Paris; AVT: Coll. A. Vigna Taglianti (Roma);

CC: Coll. A. Casale (Torino);

CD: Coll. A. Degiovanni (Bubano di Mordano, Bologna);

CM: Coll. P. Magrini (Firenze).

\section{RISULTATI}

L'esame del paratipo maschio di T. pecoudi Jeannel, 1937 ha confermato quanto era intuibile già dalla descrizione originale fornita dall'autore francese. I caratteri morfologici sia esterni (Fig. 1 e Tab. 1), sia dei genitali maschili (Figg. 3-7), dimostrano senza dubbio che l'esemplare della serie tipica di T. pecoudi Jeannel, 1937 nec pecoudi Colas \& Gaudin, 1935 (=T. montiscrystalli Casale, 1979, nomen novum) non appartiene ad alcuna specie nota delle Alpi orientali.

Al contrario, tutti i caratteri sono perfettamente coincidenti con quelli noti e descritti già da Jeannel (1921) per T. samnis del massiccio del Gran Sasso, ancora mantenuto dallo stesso Jeannel (1927) come specie valida, ma successivamente dimostrata sinonimo di T. italicus K. Daniel \& J. Daniel, 1898 da Binaghi (1959: 8), specie nota di diversi massicci montuosi dell'Appennino centrale (Magistretti, 1965; Battoni e Vigna Taglianti, 1994; Casale et al., 2006).

Di quest'ultima specie $T$. montiscrystalli è dunque sinonimo junior (cfr. in tal senso Figg. 3-5, 8-14).

\section{ACRONIMI DELLE MISURE RIPORTATE IN TABELLA 1}

L: lunghezza complessiva, dall'apice delle mandibole all'estremità delle elitre;

LT: lunghezza dal margine anteriore dell'epistoma all'estremità delle elitre;

HMW: larghezza massima del capo al rigonfiamento temporale;

LA: lunghezza delle antenne;

PL: lunghezza del pronoto, misurata lungo la linea mediana;

PMW: larghezza massima del pronoto;

PB: larghezza della base del pronoto;

EL: lunghezza elitre, misurata dalla base dell'omero all'angolo suturale;

EW: larghezza massima delle elitre;

LE: lunghezza edeago;

AN: lunghezza articolo antennale.

I preparati dei genitali raffigurati, inclusi in euparal, sono montati su acetato e allegati ai rispettivi esemplari; le macrofotografie riportate nel testo sono state eseguite da uno degli autori (PM) mediante camera digitale Nikon D2X applicata su microscopio ottico binoculare Nikon Labophot II, con obiettivi diaframmati.

Tab. 1. Misure del paratipo ổ di T. montiscrystalli Casale, 1979 (=pecoudi Jeannel, 1937).

Trechus

montiscrystalli L LT LA L LT PMW PL PB PMW EL EW EL EW HMW LE AN AN AN AN AN

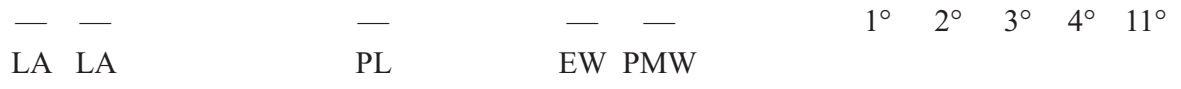

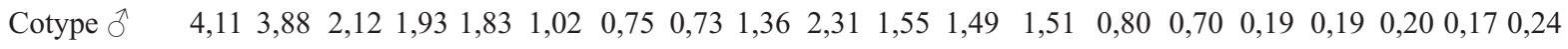



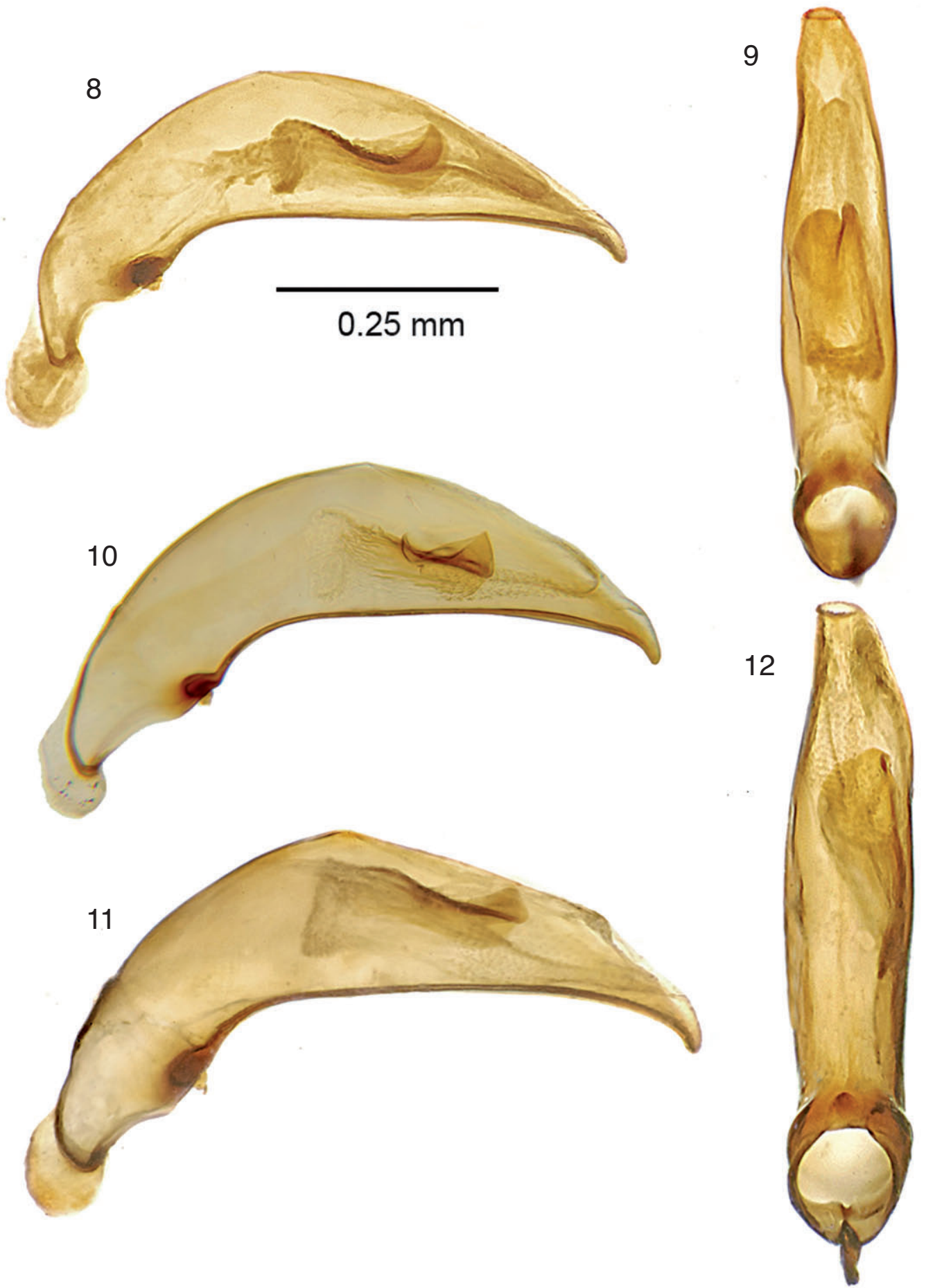

Figg. 8-12. Trechus italicus K. Daniel \& J. Daniel, 1898 (=T. samnis Jeannel, 1921). 8, Edeago in visione laterale su perspex di Gran Sasso m 2200 s.l.m. (Abruzzo) (CM); 9, ibidem in visione ventrale su perspex (CM); 10, edeago in visione laterale su acetato di Gran Sasso m 2100 s.l.m. (Abruzzo) (CD); 11, edeago in visione laterale su perspex di Monte Gorzano (Monti della Laga) m 2200 s.l.m. (Abruzzo) (CM); 12, ibidem in visione ventrale su perspex (CM). 


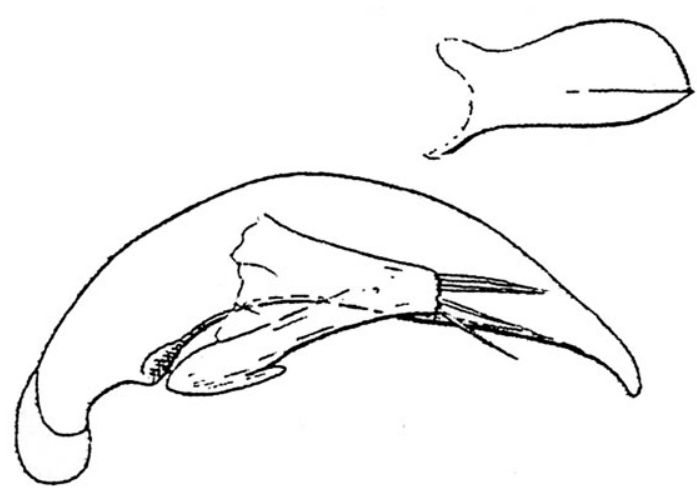

Fig. 13. Disegno originale di edeago e lamella copulatrice di Trechus pecoudi Jeannel, 1937.

\section{CONCLUSIONI}

In base ai dati acquisiti, proponiamo pertanto in questa sede la nuova sinonimia: T. montiscrystalli Casale, 1979 (=T. pecoudi Jeannel, 1937, nec pecoudi Colas \& Gaudin, 1935) = Trechus italicus K. Daniel \& J. Daniel, 1898 (=T. samnis Jeannel, 1921) nov. syn.

L'errore di Jeannel nell'attribuzione dei due esemplari della serie tipica a una località dimostratasi errata, ma così indicata nei cartellini allegati agli esemplari ("Monte Cristallo") (Fig. 2), è forse attribuibile a Jeannel medesimo, che negli anni fra il 1920 e il 1930 era intento a lavorare alla sua monumentale monografia dei Trechini mondiali, e come tale si trovava a esaminare migliaia di esemplari delle più disparate provenienze, ma più probabilmente allo stesso Pécoud, per un errore di cartellinatura di materiale.

La conferma di quest'ultima ipotesi ci viene dal fatto che in collezione Pécoud sono presenti esemplari di Carabus cavernosus variolatus O.G. Costa, 1839, con cartellino allegato "Gran Sasso, Abruzzes, Pécoud, 7.1923” (Deuve, com. pers.), cioè con data

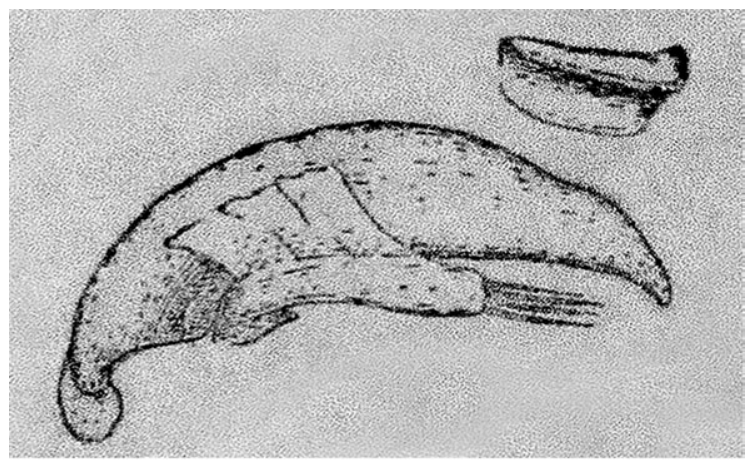

Fig. 14. Disegno originale di edeago e lamella copulatrice di Trechus samnis Jeannel, 1921.

perfettamente coincidente con quella riportata per il paratipo di T. pecoudi Jeannel esaminato (Fig. 2), a riprova che in quello stesso mese e anno Pécoud aveva raccolto materiale sul Gran Sasso d'Italia, località tipica di T. italicus.

\section{RINGRAZIAMENTI}

Per il costante supporto concesso a uno degli autori (AC) presso il MNHN di Parigi, per il prestito del paratipo di Trechus pecoudi, e per le informazioni riguardanti gli esemplari di Carabus cavernosus variolatus conservati in collezione Pécoud (MNHN), gli autori sono particolarmente grati al Dr. Thierry Deuve.

Gli autori ringraziano anche il Dr. Arnaud Faille per le preziose indicazioni sulle collezioni di Trechini in MNHN; un vivo ringraziamento è rivolto anche agli amici Augusto Degiovanni e Prof. Augusto Vigna Taglianti per averci offerto l'esame e i dati di numerosi esemplari di T. italicus di diverse località conservati nelle loro collezioni. 


\section{BIBLIOGRAFIA}

Battoni F. \& Vigna Taglianti A., 1994 - Osservazioni sui Trechus appenninici del gruppo obtusiusculus (Coleopterta, Carabidae). Fragmenta entomologica, Roma, 25 (2): 293-307.

BINAGHI G., 1959 - Contributo alla conoscenza della coleotterofauna nivale del Gran Sasso d'Italia. Bollettino della Società entomologica italiana, 89 (1-2): 6-11.

CAsale A., 1979 - Nome nuovo per un Trechus della fauna italiana. (Col. Carabidae, Trechinae). Bollettino della Società entomologica italiana, $111(4-6): 75$.

Casale A. \& Laneyrie R., 1982 - Trechodinae et Trechinae du monde. Tableau des sous-familles, tribus, séries phylétiques, genres et catalogue général des espèces. Mémoires de Biospéologie 9, 228 pp.

Casale A. \& Vigna Taglianti A., 2005 - Coleotteri Caraboidei delle Alpi e Prealpi centrali e orientali, e loro significato biogeografico (Coleoptera, Caraboidea). Biogeographia, XXVI: 129-201.

Casale A., Vigna Taglianti A., Brandmayr P. \& Colombetta G., 2006 - Insecta Coleoptera Carabidae (Carabini, Cychrini, Trechini, Abacetini, Stomini, Pterostichini). In: RufFo S., Sтосн F. (eds.), Ckmap (Checklist and distribution of the Italian fauna). Memorie del Museo Civico di Storia Naturale Verona, 2. serie, Sezione Scienze della vita, 17: 159-164, with data on CD-Rom.

Colas G. \& Gaudin G., 1935 - Sur de nouveaux Trechinae des Pyrénées occidentales. Revue française d'Entomologie, 1: 245 253.

Coulon J., Pupier R., Quérnnec É., Ollivier É. \& Richoux P., 2011 - Coléoptères Carabiques. Compléments aux deux volumes de René Jeannel, mises à jour, corrections et répertoire. Faune de France 94 (vol. 1): 1 - 352, planches I-XII.

JEANNEl R., 1921 - Notes sur les Trechini (Coleopt. Carabidae). Buletinul Societăţii de Stiinţe din Cluj, 1 (1921-1923): 154170.

JEAnNEl R., 1927 - Monographie des Trechinae 2. Morphologie comparée et distribution géographique d'un groupe de Coléoptères. L'Abeille, Paris, 33: 1-592.

JEANNEL R., 1937 - Nouveaux Trechinae paléarctiques. Bulletin de la Société entomologique de France, 42 : 82-88.

Löbl I. \& SmetanA A., 2003 - Catalogue of Palearctic Coleoptera. Vol. 1. Archostemata - Myxophaga - Adephaga. Apollo Books, Stenstrup, 819 pp.

LoRenz W., 2005 - Systematic list of extant ground beetles of the world (Insecta Coleoptera "Geadephaga": Trachypachidae and Carabidae. incl. Paussinae, Cicindelinae, Rhysodinae). Tutzing, iv $+530 \mathrm{pp}$.

Magistretti M., 1965 - Fauna d'Italia, VIII. Coleoptera: Cicindelidae, Carabidae. Catalogo topografico. Calderini, Bologna, $512 \mathrm{pp}$.

Vigna taglianti A., 2005 - Checklist e corotipi delle specie di Carabidae della fauna italiana. Appendice B. pp. 186-225. In: Brandmayr P., Zetto T. \& Pizzolotto R. (eds.), I Coleotteri Carabidi per la valutazione ambientale e la conservazione della biodiversità. Manuale operativo. APAT, Manuali e Linee Guida, 34: 240 pp.

Indirizzo degli autori:

Achille Casale, Università di Sassari, Dipartimento di Scienze della Natura e del Territorio, Sezione Zoologia, via Muroni 25, 07100 Sassari, Italia. E-mail: a_casale@libero.it; casale@uniss.it

Paolo Magrini, Museo di Storia Naturale dell’Università di Firenze, Sezione di Zoologia "La Specola", via Romana 17, 50125 Firenze, Italia. E-mail: duvalius@paolomagrini.it 